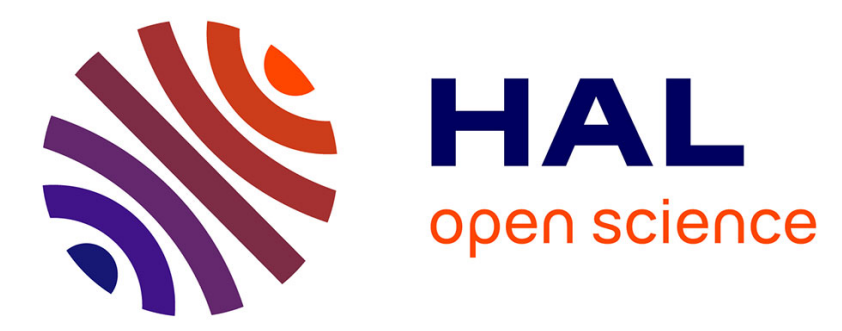

\title{
Permeability fluctuations in heterogeneous networks with different dimensionality and topology
}

Yves Bernabé, Céline Bruderer-Weng, Alexis Maineult

\section{To cite this version:}

Yves Bernabé, Céline Bruderer-Weng, Alexis Maineult. Permeability fluctuations in heterogeneous networks with different dimensionality and topology. Journal of Geophysical Research: Solid Earth, 2003, 108 (B7), pp.2351. 10.1029/2002JB002326 . insu-01729212

\section{HAL Id: insu-01729212 \\ https://hal-insu.archives-ouvertes.fr/insu-01729212}

Submitted on 12 Mar 2018

HAL is a multi-disciplinary open access archive for the deposit and dissemination of scientific research documents, whether they are published or not. The documents may come from teaching and research institutions in France or abroad, or from public or private research centers.
L'archive ouverte pluridisciplinaire HAL, est destinée au dépôt et à la diffusion de documents scientifiques de niveau recherche, publiés ou non, émanant des établissements d'enseignement et de recherche français ou étrangers, des laboratoires publics ou privés. 


\title{
Permeability fluctuations in heterogeneous networks with different dimensionality and topology
}

\author{
Yves Bernabé \\ Institut de Physique du Globe, Université Louis Pasteur, Centre National de Recherche Scientifique, Strasbourg, France \\ Céline Bruderer-Weng \\ Hydrosciences, Institut des Sciences de la Terre, de l'Environnement et de l'Espace de Montpellier, Université de Montpellier \\ 2, Montpellier, France
}

\begin{abstract}
Alexis Maineult
Institut de Physique du Globe, Université Louis Pasteur, Centre National de Recherche Scientifique, Strasbourg, France

Received 27 November 2002; revised 3 April 2003; accepted 21 April 2003; published 29 July 2003.

[1] The purpose of this work was to relate the spatial fluctuations and scaling properties of the transport properties of porous rocks to their underlying pore geometry. Our approach was to numerically simulate flow through networks of pipes with randomly prescribed radii. The permeability $k$ and inverse formation factor $1 / F$ were calculated in a large number of network realizations of varying size and degree of heterogeneity (i.e., the width of the pipe radius distribution). We generally observed a large decrease of the ensemble arithmetic averages of $k$ and $1 / F$ with increasing network size (i.e., negative scale effect). Conversely, the ensemble geometric averages showed a moderate positive scale effect in three-dimensional simple cubic networks. We also found that in networks smaller than $32 \times 32$ or $10 \times 10 \times 10$, the ensemble standard deviations of $k$ and $1 / F$ had a power law dependence on network size (defined as the total number of pipes) with an exponent $\alpha$ varying from -0.5 in homogeneous networks to large negative values depending on lattice topology in highly heterogeneous ones $(-\alpha$ increased with increasing lattice connectiveness, i.e., with coordination number). Thus at small scales the network transport properties were characterized by a nonuniversal power law scaling. At larger scales we observed a transition to a presumably "universal" power law scaling with an exponent equal to -0.5 independently on the degree of heterogeneity, dimensionality and lattice topology. Comparing our results to published experimental data, we found a good agreement, except in cases where we suspect that the small-scale measurements suffered a significant bias (indicated by non-nested distributions at increasing scales). We speculate that the strong positive scale effect generally observed in nature is also caused by sampling bias at small scales. INDEX TERMS: 1829 Hydrology: Groundwater hydrology; 5114 Physical Properties of Rocks: Permeability and porosity; 5139 Physical Properties of Rocks: Transport properties; KEYWORDS: porosity and permeability fluctuations, effective rock properties, heterogeneous media
\end{abstract}

Citation: Bernabé, Y., C. Bruderer-Weng, and A. Maineult, Permeability fluctuations in heterogeneous networks with different dimensionality and topology, J. Geophys. Res., 108(B7), 2351, doi:10.1029/2002JB002326, 2003.

\section{Introduction}

[2] Developments in hydrology over the last two decades have amply demonstrated that geologic materials have extremely variable transport properties at all observable scales [e.g., Gelhar, 1993; Sahimi, 1995, and references therein]. This high level of heterogeneity curtails effective prediction of aquifer behavior because a sufficient amount of detailed information cannot be possibly collected. Rock permeability is often measured in sampling volumes much smaller than the grid blocks used in numeric flow simu-

Copyright 2003 by the American Geophysical Union. 0148-0227/03/2002JB002326\$09.00 lators, and, hence, must be upscaled [e.g., Durlofsky, 1991, 1992; Renard and de Marsily, 1997]. This is a difficult problem because permeability does not scale according to a simple averaging rule [e.g., Cushman, 1986; Dagan, 1986; Newman, 1994]. Transport properties upscaling has been investigated by simulating flow through numerically generated random porous media, assuming a specific (usually lognormal) permeability distribution [e.g., Gelhar and Axness, 1983; Ababou et al., 1989]. Concurrently, efforts have been expended to estimate the scaling properties and the distribution of permeability in geomaterials and geologic formations [e.g., Brace, 1980; Goggin et al., 1992; Garbesi et al., 1996; Painter, 1996; Schulze-Makuch et al., 1999]. Recent laboratory studies [Henriette et al., 1989; Tidwell 
and Wilson, 1997, 1999a, 1999b, 2000] attempted to quantify the spatial fluctuations of permeability in meter-scale blocks as a function of measurement volume (see also Zhang et al. [2000] who examined centimeter-scale samples). Similarly, the spatial fluctuations of porosity and other pore geometry parameters were evaluated at the laboratory scale [Henriette et al., 1989; Brown et al., 2000; Zhang et al., 2000]. Pore-scale microstructure observations generally reveal very broad and skewed distributions of pore sizes or fissure apertures [Wong et al., 1989; Netto, 1993; Fredrich and Lindquist, 1997; Zhu and Wong, 1999; Lindquist et al., 2000].

[3] The purpose of this work is to relate the spatial fluctuations and scaling properties of permeability to their underlying pore geometry. Our approach is based on numerically simulating flow in heterogeneous pipe networks while systematically varying the degree of heterogeneity (i.e., the width of the pipe radius distribution) and the network size. Since permeability and other transport properties are controlled by the percolation properties of the pore network [Sahimi, 1995], we also investigated the influence of dimensionality (that is, we used two-dimensional and three-dimensional networks) and lattice topology (that is, we compared results obtained in two-dimensional square, triangular and hexagonal networks). In order to gain deeper insight, we performed a similar study of the electrical formation factor (i.e., the ratio of fluid bulk conductivity to rock conductivity in absence of surface conduction effects). This last aspect is interesting in its own right because estimating permeability from electrical conductivity measurements is often attempted in the field [e.g., Purvance and Andricevic, 2000a, 2000b]. Note that, in this problem, the fluid ionic strength and, therefore, its electrical conductivity are usually assumed constant over the entire system considered. This assumption may not hold in all field situations (see Hunt [2001] for a theoretical treatment of the variable ionic strength case).

\section{Numerical Procedures}

[4] The numerical procedure followed in this work consists in three principal steps:

[5] (1) Construct a large number of random network realizations (i.e., up to 100,000 for small network sizes and no less than 1000, except for the largest networks that we solved using a CPU-time demanding, iterative method. In the most unfavorable cases we were limited to 350 realizations).

[6] (2) Calculate their permeability $k$ and inverse formation factor $1 / F$ by solving the appropriate system of linear equations [e.g., Bernabé and Bruderer, 1998, and references therein]. We used periodic boundary conditions and, therefore, were able to calculate the full permeability and electrical conductivity tensors (however, in the rest of the paper, $k$ and $1 / F$ will solely denote the diagonal components).

[7] (3) Analyze the resulting $k$ and $1 / F$ ensemble distributions. Although complete information is theoretically available, we limited the analysis to the first two moments.

[8] We systematically and independently varied the degree of heterogeneity and the size of the network realizations in order to observe how these two parameters affect the resulting $k$ and $1 / F$ distributions. We also investigated the influence of dimensionality and topology by using twodimensional (2D) square, triangular and hexagonal lattices as well as 3D simple cubic lattices. Notice that we completed the characterization of the network realizations by calculating other relevant, secondary parameters such as porosity $\Phi$ or critical radius $r_{c}$ (i.e., the pipe radius corresponding to disconnection of the network [e.g., Bernabé and Bruderer, 1998; Friedman and Seaton, 1998]).

[9] One important parameter to define is the "degree of heterogeneity." Many definitions have been used in the literature (not always univocally related to each other). Here, we followed our previous work [Bernabé and Bruderer, 1998; Bruderer and Bernabé, 2001] and measured heterogeneity as the normalized standard deviation $\sigma_{r} /\langle r\rangle$, where $\sigma_{r}$ denotes the standard deviation and $\langle r\rangle$ the mean of the pipe radius distribution. We also need to clarify what we mean by network size. In order to unify the statistical analysis of our results for different lattices, it is best to use the number of pipes, $n$, in each network realization. The number of nodes or the linear spatial dimension $S$ (i.e., scale) of the network realizations can be easily derived from $n$ ( $n$ is proportional to $S^{2}$ and $S^{3}$ in twodimensional and three-dimensional lattices, respectively). Since we used periodic boundary conditions, all nodes are connected to the same number of pipes. Hence $n$ is equal to the total number of nodes times the coordination number $Z$ divided by 2 . The smallest networks used here were $3 \times 3$ ( $n=18$ for the square lattice) and $3 \times 3 \times 3(n=81)$. When we used the Cholesky decomposition method (ChD) for computing $k$ and $1 / F$, the largest networks were $32 \times 32(n$ $=2048$ for the square lattice $)$ and $10 \times 10 \times 10(n=3000)$ whereas they were $192 \times 192(n=73728)$ and $30 \times 30 \times$ $30(n=81,000)$ when we utilized the preconditioned biconjugate gradient method (PBCG). The $\mathrm{ChD}$ method [Press et al., 1992] is very efficient and accurate even in the most heterogeneous cases considered here, but requires an overly large amount of memory space when the network size exceeds the limit mentioned above. The PBCG method [Press et al., 1992] is a fast and efficient iterative optimization method, much less demanding in memory space than ChD. However, its accuracy and convergence rate decrease with network size and heterogeneity level, which leads to a serious problem. For moderately large $\sigma_{r} /\langle r\rangle$ and $n$, "pathological" realizations occur, for which the method fails to converge within the maximum number of iterations allowed (i.e., here one million). Simply discarding these "pathological" realizations is not a good option since it will produce a bias in the resulting ensemble statistics. Alternatively, one can relax the arrest condition but this diminishes the accuracy and, in fact, does not always succeed. For this reason the results obtained with these two methods are not equivalent statistically and will be discussed separately in sections 3 and 4 . Notice that we verified that the two methods gave essentially identical results in $30 \times 30$ and $10 \times 10 \times 10$ networks.

[10] Before describing the various pipe radius distributions (or $r$ distributions) used here, we point out that this study was restricted to statistically isotropic networks with uncorrelated spatial distributions of pipe radii. We realize that these simplifying assumptions may be rarely satisfied in geological materials, but investigating simple cases is an important preliminary step toward understanding much 
Table 1. Parameters of the $r$ Distributions Used in This Work

\begin{tabular}{lcccccc}
\hline & \multicolumn{5}{c}{$\sigma_{r} /\langle r\rangle$} \\
\cline { 2 - 6 } & 0.06 & 0.4 & 0.8 & 1.2 & 1.6 \\
\hline A, 2D square, $\left[r_{\min }, r_{\max }\right]$ & {$[0.91,1.1]$} & {$[0.5,2]$.} & {$[0.25,4]$.} & {$[0.091,11]$.} & {$[0.032,31]$.} & {$[0.0099,101]$.} \\
B, 3D simple cubic, $\left[r_{\min }, r_{\max }\right]$ & {$[0.867,1.05]$} & {$[0.354,1.41]$} & {$[0.125,2]$.} & {$[0.0274,3.32]$} & {$[0.00574,5.57]$} & {$[0.000985,10.05]$} \\
C, 2D triangular, $\left[r_{\min }, r_{\max }\right]$ & {$[0.883,1.07]$} & {$[0.406,1.62]$} & {$[0.165,2.64]$} & {$[0.0443,5.36]$} & {$[0.0115,11.1]$} & {$[0.00248,25.3]$} \\
D, 2D hexagonal lattices, $\left[r_{\min }, r_{\max }\right]$ & {$[0.935,1.13]$} & {$[0.616,2.46]$} & {$[0.379,6.06]$} & {$[0.187,22.6]$} & {$[0.0904,86.9]$} & {$[0.0395,403]$.} \\
\hline
\end{tabular}

more complex problems. Furthermore, results obtained from uncorrelated spatial distributions were found to remain approximately valid in isotropic systems at scales larger than the correlation length [e.g., Hunt, 1998].

\subsection{Two-Dimensional Square Networks}

[11] We first focus on the case of the 2D square lattice. This type of lattice is by far the most commonly used in network simulation studies but, as we will try to demonstrate, it has an exceptional behavior owing to the symmetry of its bond percolation threshold (i.e., the median of the pipe radius distribution [Stauffer and Aharony, 1992]). As we will show later, lattices with asymmetrical percolation thresholds display less regularities in their behavior.

[12] We used $\log$-uniform $r$ distributions, i.e., $\log (r)$ uniformly distributed between $\log \left(r_{\text {min }}\right)$ and $\log \left(r_{\text {max }}\right)$. This type of distribution is less realistic than the lognormal distribution but has similar basic properties (i.e., skewness) and is much less costly computationally, a crucial feature given the very large number of realizations constructed here. We selected $r_{\min }$ and $r_{\max }$ in such a way as to vary $\sigma_{r} /\langle r\rangle$ while keeping the median $m=r_{\min }^{0.5} r_{\max }^{0.5}$ constant, equal to unity. In the present work, we used 6 different intervals $\left[r_{\min }, r_{\max }\right]$ corresponding to increasing values of $\sigma_{r} /\langle r\rangle$ (see Table 1). These 6 distributions are labeled $\mathbf{A}$ in the rest of the paper. The range of $\sigma_{r} /\langle r\rangle$ investigated is very broad, namely 0.06 for $\mathbf{A 1}, 0.4$ for $\mathbf{A 2}, 0.8$ for $\mathbf{A} 3,1.2$ for A4, 1.6 for A5 and 1.9 for A6. The distribution A1 corresponds to near homogeneity and A6 to an extremely large heterogeneity with a normalized standard deviation of $\log _{\mathrm{e}}(k)$ greater than 8 .

\subsection{Three-Dimensional Simple Cubic and Two- Dimensional Triangular and Hexagonal Networks}

[13] For comparison purpose, we started by simply applying the same 6 distributions $\mathbf{A}$ as above. However, the critical path analysis based on percolation theory [Ambegaokar et al., 1971; Pollak, 1972] shows that the transport properties of heterogeneous networks are controlled by the critical radius $r_{c}$ through the following equation, $r_{c}{ }^{2}=8 k F$ [e.g., Bernabé and Bruderer, 1998; Friedman and Seaton, 1998]. In infinitely large networks, $r_{c}$ is given by the quantile corresponding to the percolation threshold of each particular lattice (i.e., the upper quartile for the simple cubic lattice, and, the 0.65-quantiles and 0.35-quantiles for the triangular and hexagonal lattices, respectively). Consequently, in the case of distributions A, $r_{c}$ varies when $\sigma_{r} /\langle r\rangle$ is changed, resulting in systematic variations of $k$ and $1 / F$. To remove this extra degree of freedom, we additionally tested $r$ distributions specific to each type of lattice, in which the appropriate quantiles, and hence $r_{c}$, were kept equal to unity, while still producing the same values of $\sigma_{r} /\langle r\rangle$ as above. These additional distributions are labeled $\mathbf{B}, \mathbf{C}$ and $\mathbf{D}$, corresponding to $3 \mathrm{D}$ simple cubic, 2D triangular and 2D hexagonal lattices, respectively. The corresponding intervals $\left[r_{\min }, r_{\max }\right]$ are reported in Table 1.

\section{Results}

\subsection{Two-Dimensional Square Networks}

[14] Again, we find it most convenient to begin by a full description of our results in the square lattice case. First of all, we report some assessment details about the computing methods. We observed that $\mathrm{ChD}$ performed equally well with all $6 \mathrm{~A}$ distributions. On the other hand, PBCG only worked optimally in the $\mathbf{A} \mathbf{1}, \mathbf{A} \mathbf{2}$ and $\mathbf{A} \mathbf{3}$ cases. In the hydraulic case and for the distributions A4 and A5, we were able to limit the percentage of unsolved realizations to a few percents by reducing the mean-square residual criterion to $10^{-10}$ and $10^{-8}$, respectively. For A6 we obtained about $50 \%$ of failures in $30 \times 30$ networks after further reducing the criterion to $10^{-6}$ and close to $100 \%$ in larger networks. We encountered much less difficulties with the $1 / F$ calculations. The reason is that the individual electric conductances cover a much narrower range than the hydraulic conductances (indeed, they depend on $r^{2}$ while the hydraulic conductances vary as $r^{4}$ ).

[15] Let us denote $k_{o}$ and $1 / F_{o}$ the permeability and inverse formation factor of a perfectly homogeneous network with unit pipe radius. Since $k_{o}$ and $1 / F_{o}$ are obviously independent on $n$, it is appropriate, in the rest of the paper, to normalize $k$ and $1 / F$ with respect to $k_{o}$ and $1 / F_{o}$. Using this normalization convention, we first observe that, independently on $\sigma_{r} /\langle r\rangle, k$ and $1 / F$ both approach unity as $n$ increases (see Figure 1a). In other words, for all degrees of heterogeneity, we can write $k_{\infty}=1 / F_{\infty}=1$, where the subscript $\infty$ refers to an infinite network. As we will see later this property is unique to the $2 \mathrm{D}$ square lattice.

[16] A second observation is that $\langle k\rangle_{a}$, the ensemble arithmetic mean of $k$, strongly increases with decreasing $n$ and increasing $\sigma_{r} /\langle r\rangle$ (see Figure 1a), whereas $\langle k\rangle_{g}$, the ensemble geometric mean, remains equal to unity (within numerical precision) for all network sizes and degrees of heterogeneity (not shown in Figure 1). Again, this last property is unique to the square lattice. The inverse formation factor exhibited the same properties, except that $\langle 1 / F\rangle_{a}$ varied much less sharply than $\langle k\rangle_{a}$. In order to establish a quantitative scaling relation [Hunt, 1998, 2001], we have to consider the normalized difference $\delta\left(\langle k\rangle_{a}\right)=\left(\langle k\rangle_{a}-k_{\infty}\right) / k_{\infty}$ (in the square lattice case the normalization is not necessary since $k_{\infty}=1 / F_{\infty}=\langle k\rangle_{g}=\langle 1 / F\rangle_{g}=1$, but, as will be shown later, it is mandatory in the other lattices). We plotted $\delta\left(\langle k\rangle_{a}\right)$ against $n$ for various values of $\sigma_{r} /\langle r\rangle$ in log-log scale in Figure $1 \mathrm{~b}$. For each value of $\sigma_{r} /\langle r\rangle$ and within the range of 

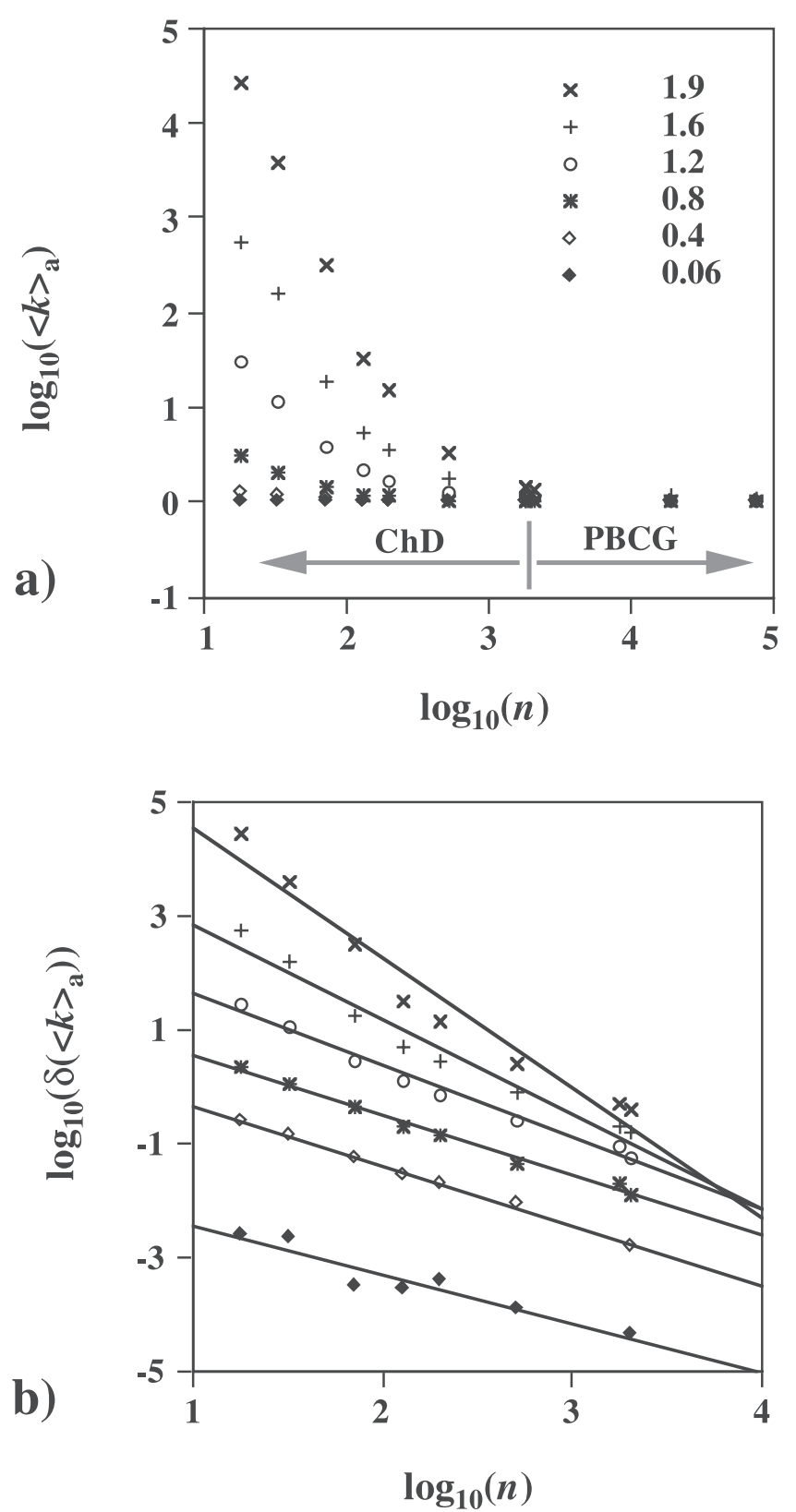

Figure 1. Illustration of the permeability scaling in the case of the 2D square lattice. (a) Dependence of $\langle k\rangle_{a}$ on network size $n$. As indicated in the diagram, the $\mathrm{ChD}$ solving method was used in networks smaller than $32 \times 32$ while PBCG was utilized above this size. (b) The power law dependence of $\delta\left(\langle k\rangle_{a}\right)=\left(\langle k\rangle_{a}-k_{\infty}\right) / k_{\infty}$ on network size $n$. Note that only the $\mathrm{ChD}$ results are included in this analysis. The symbols correspond to the values of $\sigma_{r} /\langle r\rangle$ as indicated in the upper right corner.

$n$ investigated, $\delta\left(\langle k\rangle_{a}\right)$ shows a distinct power law dependence on $n$, i.e., $\log _{10}\left[\delta\left(\langle k\rangle_{a}\right)\right]=\alpha \log _{10}[n]+\beta$, where $\alpha$ denotes the exponent and $\beta$ the decimal logarithm of the prefactor. Likewise, $\delta\left(\langle 1 / F\rangle_{a}\right)$ had a similar behavior. For both $\delta\left(\langle k\rangle_{a}\right)$ and $\delta\left(\langle 1 / F\rangle_{a}\right),-\alpha$ and $\beta$ increase with increasing $\sigma_{r} /\langle r\rangle$ although the variations are much smaller for $\delta\left(\langle 1 / F\rangle_{a}\right)$ (see Figures 2 and 3 ). In particular, the exponent $\alpha$ approaches -1 for homogeneous networks. Note that the power law analysis described above was limited to networks smaller than $32 \times 32$. The reason for this was that $\delta\left(\langle k\rangle_{a}\right)$ and $\delta\left(\langle 1 / F\rangle_{a}\right)$ in larger networks became smaller than the finite ensemble statistical noise (negative values of $\delta\left(\langle k\rangle_{a}\right)$ and $\delta\left(\langle 1 / F\rangle_{a}\right)$ can even occur). However, we do not believe that the power laws reported above can be extrapolated to
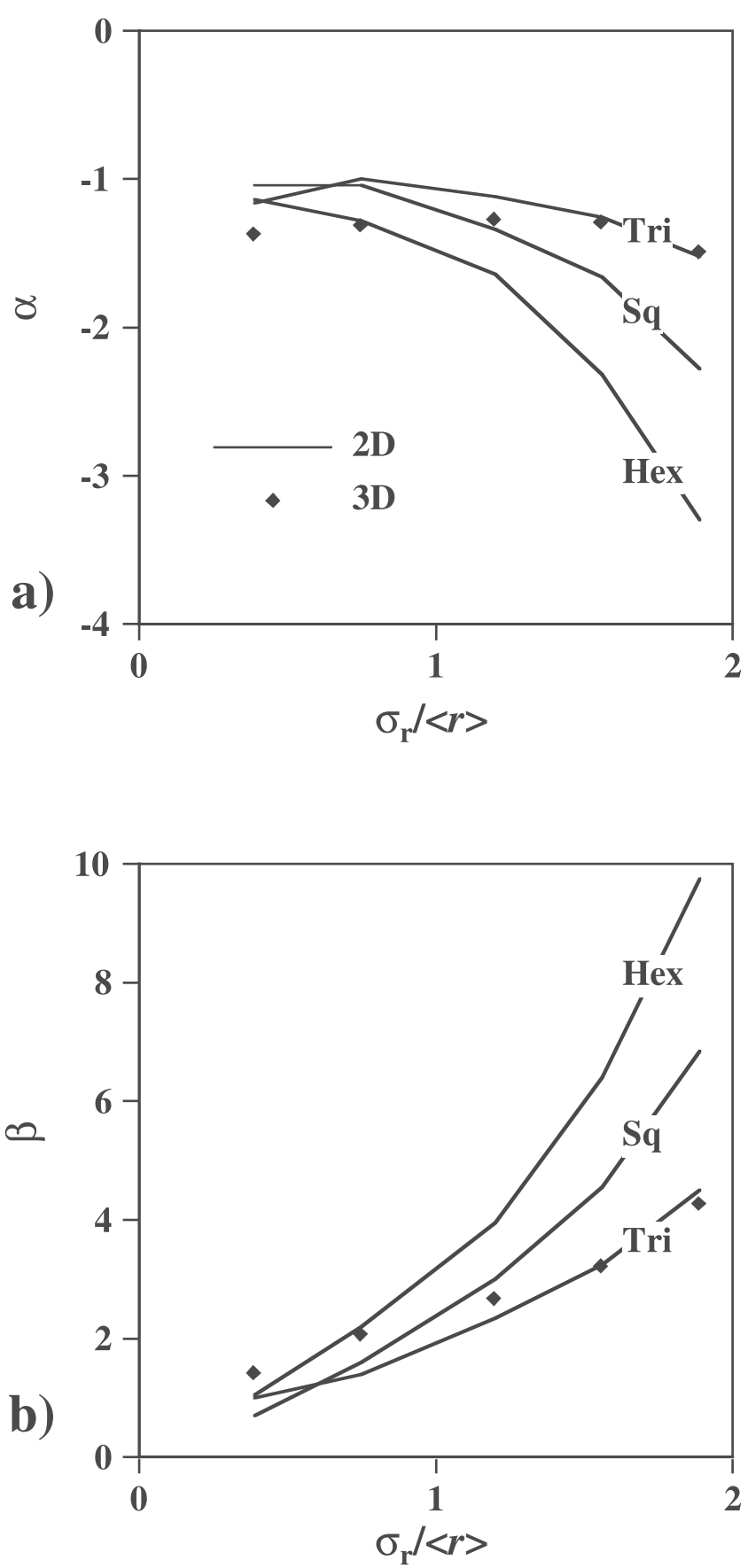

Figure 2. The power law parameters calculated for $\delta\left(\langle k\rangle_{a}\right)$ using the $\mathrm{ChD}$ results only (i.e., for networks smaller than $32 \times 32$ or $10 \times 10 \times 10$ ): (a) the exponent $\alpha$ and (b) $\beta$, the decimal log of the pre-factor. The solid lines correspond to the 2D lattices, the letters Tri, Sq and Hex indicating the triangular, square and hexagonal lattices, respectively. The diamonds represent the results for the 3D simple cubic lattice. 

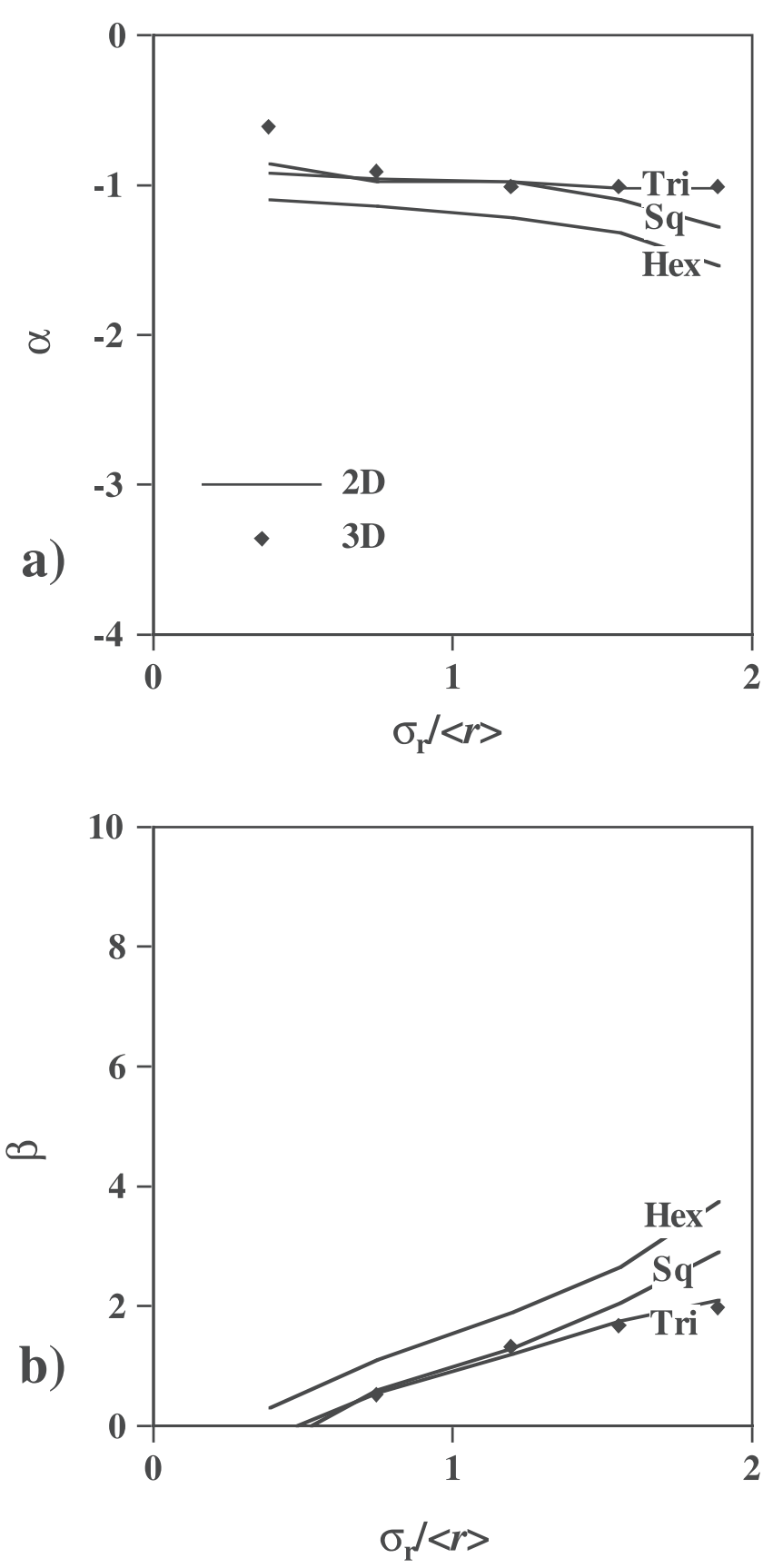

Figure 3. The power law parameters calculated for $\delta(\langle 1 /$ $F\rangle_{a}$ ) using the $\mathrm{ChD}$ results only (i.e., for networks smaller than $32 \times 32$ or $10 \times 10 \times 10$ ): (a) the exponent $\alpha$ and (b) $\beta$, the decimal log of the pre-factor. The symbols are the same as in Figure 2.

infinite size $n$ (see discussion in section 4). We attempted to perform the same analysis on $\delta\left(\langle k\rangle_{g}\right)$ and $\delta\left(\langle 1 / F\rangle_{g}\right)$, but, because $\langle k\rangle_{g}$ and $\langle 1 / F\rangle_{g} \approx 1$ (i.e., $\delta\left(\langle k\rangle_{g}\right)$ and $\delta\left(\langle 1 / F\rangle_{g}\right) \approx 0$ ) for all values of $n$, the data appeared to be essentially noise. As we will see later, $\delta\left(\langle k\rangle_{g}\right)$ and $\delta\left(\langle 1 / F\rangle_{g}\right)$ do show power law dependence on $n$ in the other lattices.

[17] A third observation concerns the ensemble standard deviations of $k$ and $1 / F, \sigma_{k}$ and $\sigma_{1 / F}$. As expected, $\sigma_{k}$ and $\sigma_{1 / F}$ strongly decrease with increasing $n$. Consistently with the other observations, the variations of $\sigma_{1 / F}$ are less dramatic than those of $\sigma_{k}$. The values of $\sigma_{k}$ obtained using $\mathrm{ChD}$ (i.e., for networks up to $32 \times 32$ ) are plotted in Figure 4a against $n$ in $\log -\log$ scale, revealing a clear power law behavior. The fit quality is better for $\sigma_{k}$ than it was in Figure $1 \mathrm{~b}$ for $\delta\left(\langle k\rangle_{a}\right)$, indicating that the statistical noise in the determination of $\sigma_{k}$ is low. Similar observations can be made with $\sigma_{1 / F}$. Again, the values of $-\alpha$ and $\beta$ calculated for both $\sigma_{k}$ and $\sigma_{1 / F}$ increase with increasing $\sigma_{r} /\langle r\rangle$ (see Figures 5 and 6). This time, the exponent $\alpha$ is approximately equal to -0.5 for homogeneous networks. The values of $\sigma_{k}$ obtained using PBCG (i.e., for networks $30 \times 30$ and larger) are plotted in Figure $4 \mathrm{~b}$. We can see that these large network data suggest a transition from the previously described power law behavior to another one independent on $\sigma_{r} /\langle r\rangle$ (i.e., universal), characterized by $\alpha=-0.5$.

[18] The results described above only concern the diagonal components of the $k$ and $1 / F$ tensors. However, the individual realizations, despite having been isotropically generated, all displayed some degree of anisotropy. Inspection of the nondiagonal components of the $k$ and $1 / F$ tensors showed that their ensemble distributions had near-zero means. Their ensemble standard deviations were much smaller than those of the diagonal components, and decreased in a power law fashion with increasing network size (the exponent was closer to -0.5 in this case than for $\sigma_{k}$ and $\left.\sigma_{1 / F}\right)$. This demonstrates that the network realizations generated were indeed statistically isotropic (note that the statistical deviations from isotropy were much lower for $1 / F$ than $k$ ). Statistical isotropy was also verified in the other lattices and will not be discussed further in this article.

[19] Each individual network realization corresponds to a set of values of $r$ (called an $r$-set). Statistical parameters such as the median $m$ or the second moment $\left\langle r^{2}\right\rangle$ (notice that $\left\langle r^{2}\right\rangle$ is proportional to the porosity $\left.\Phi\right)$ can be calculated on these $r$-sets. We know that the ensemble standard deviation of such parameters must be proportional to the inverse square root of the $r$-set size (i.e., $n$ ). We indeed verified that practically exact power laws with $\alpha=-0.5$ were obtained for $\sigma_{m}$ and $\sigma_{\Phi}$. Obviously, this property is independent on lattice dimensionality and topology.

[20] Finally, we determined the critical radius $r_{c}$ for each network realization. Although it must be equal to $m$ in infinite networks, $r_{c}$ is not a simple statistical parameter of the $r$-sets. For example, consider an arbitrary network realization and imagine that we create another one by redistributing identical pipes in different locations. The new realization has an identical $r$-set but a possibly very different $r_{c}$. The behavior of $r_{c}$ appeared to be similar to that of $k$ and $1 / F$. We will not describe it in detail here, but, instead, emphasize the striking observation that the relationship $r_{c}^{2}=k F$ (the factor 8 is removed here because of the normalization) was satisfied within better than $20 \%$ in all individual network realizations, independently on size or degree of heterogeneity. This extremely important (nonstatistical) property was observed to be also true in the other 2D lattices, suggesting that the discussion in Bernabé [1997] linking $r_{c}$ to the critical frequency at the viscousinertial transition [see also Bernabé and Bruderer, 1998] is generally valid for $2 \mathrm{D}$ lattices. In the case of the $3 \mathrm{D}$ simple cubic lattice we found a slight discrepancy (namely, $k F$ $\approx 0.7 r_{c}^{2}$ ). The cause of this discrepancy is unclear and will not be discussed further in the present paper (for a theoret- 
a)
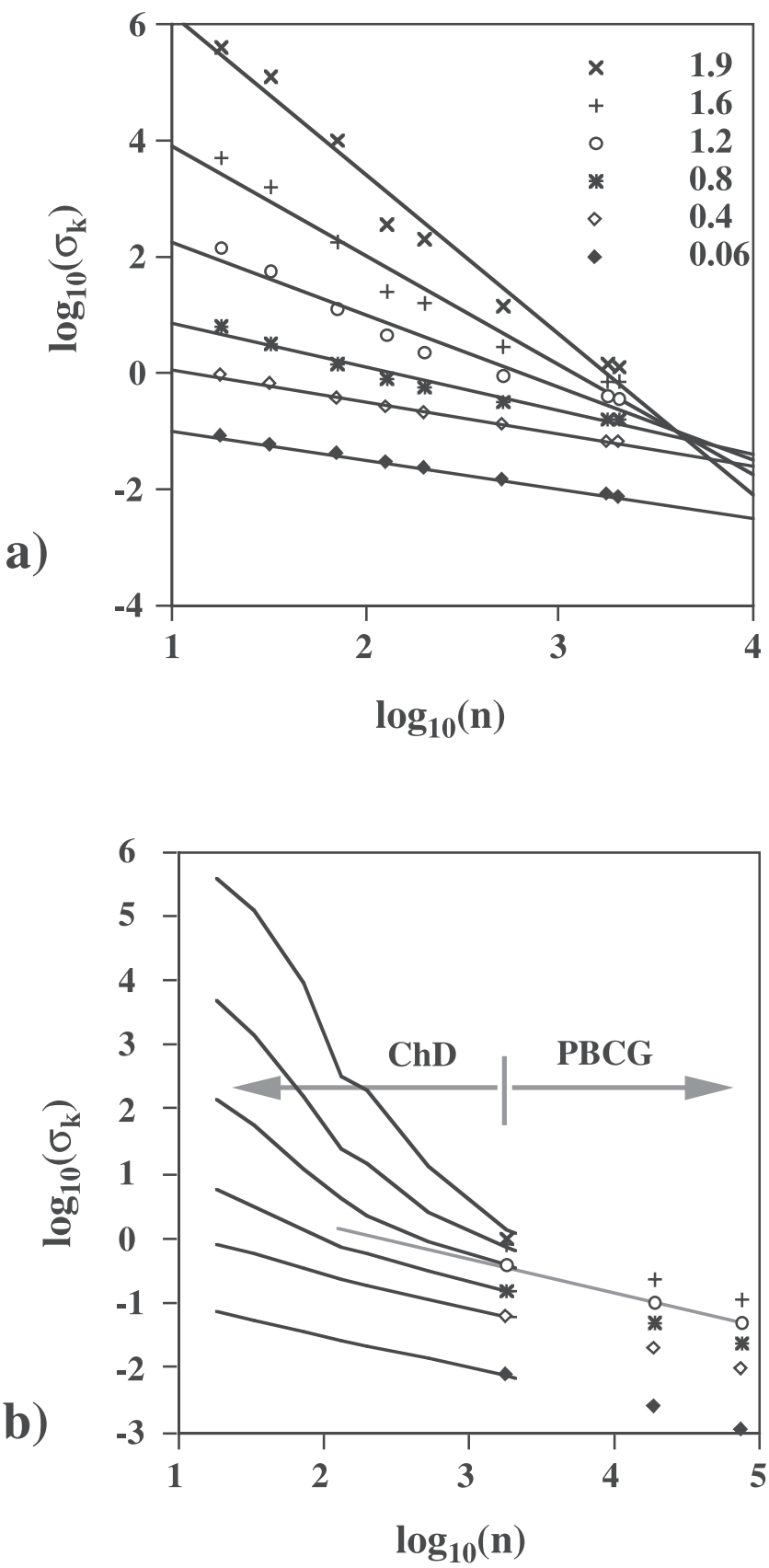

Figure 4. Illustration of the permeability fluctuations scaling in the case of the $2 \mathrm{D}$ square lattice. (a) The power law dependence of $\sigma_{k}$ on network size $n$. Note that only the $\mathrm{ChD}$ results are included in this analysis (i.e., networks smaller than $32 \times 32$ ). The symbols correspond to the values of $\sigma_{r} /\langle r\rangle$ as indicated in the upper right corner. (b) Dependence of $\sigma_{k}$ on network size $n$ in the entire range of $n$. The ChD results already shown in Figure 4a are now represented by solid lines while the PBCG data points are plotted using the same symbols as in Figure 4a. The shaded line superposed on the open circle points indicates the -0.5 slope and helps in visualizing the transition from nonuniversal to universal scaling (see text).
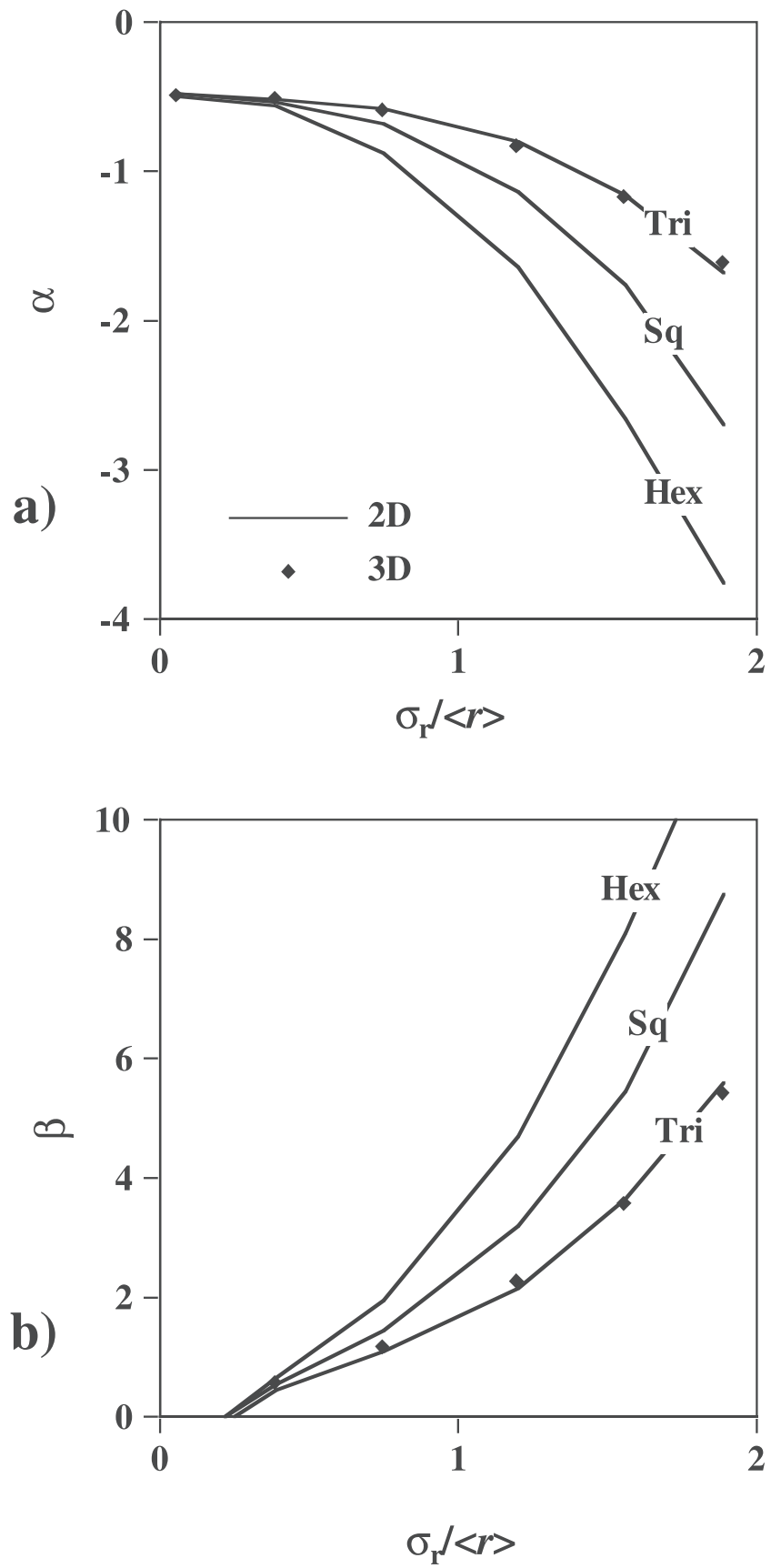

Figure 5. The power law parameters calculated for $\sigma_{k}$ using the $\mathrm{ChD}$ results only (i.e., for networks smaller than $32 \times 32$ or $10 \times 10 \times 10$ ): (a) the exponent $\alpha$ and (b) $\beta$, the decimal log of the pre-factor. The symbols are the same as in Figure 2.

ical discussion of $r_{c}$ based on percolation cluster statistics, see Hunt [2001]).

\subsection{Three-Dimensional Simple Cubic and Two- Dimensional Triangular and Hexagonal Networks}

[21] First of all, it is interesting to note that the PBCG solving method was more successful in $3 \mathrm{D}$ simple cubic networks than in 2D square ones. There were almost no unsolved realizations except with distribution B6 for which we reduced the mean-square residual criterion to $10^{-10}$. For 

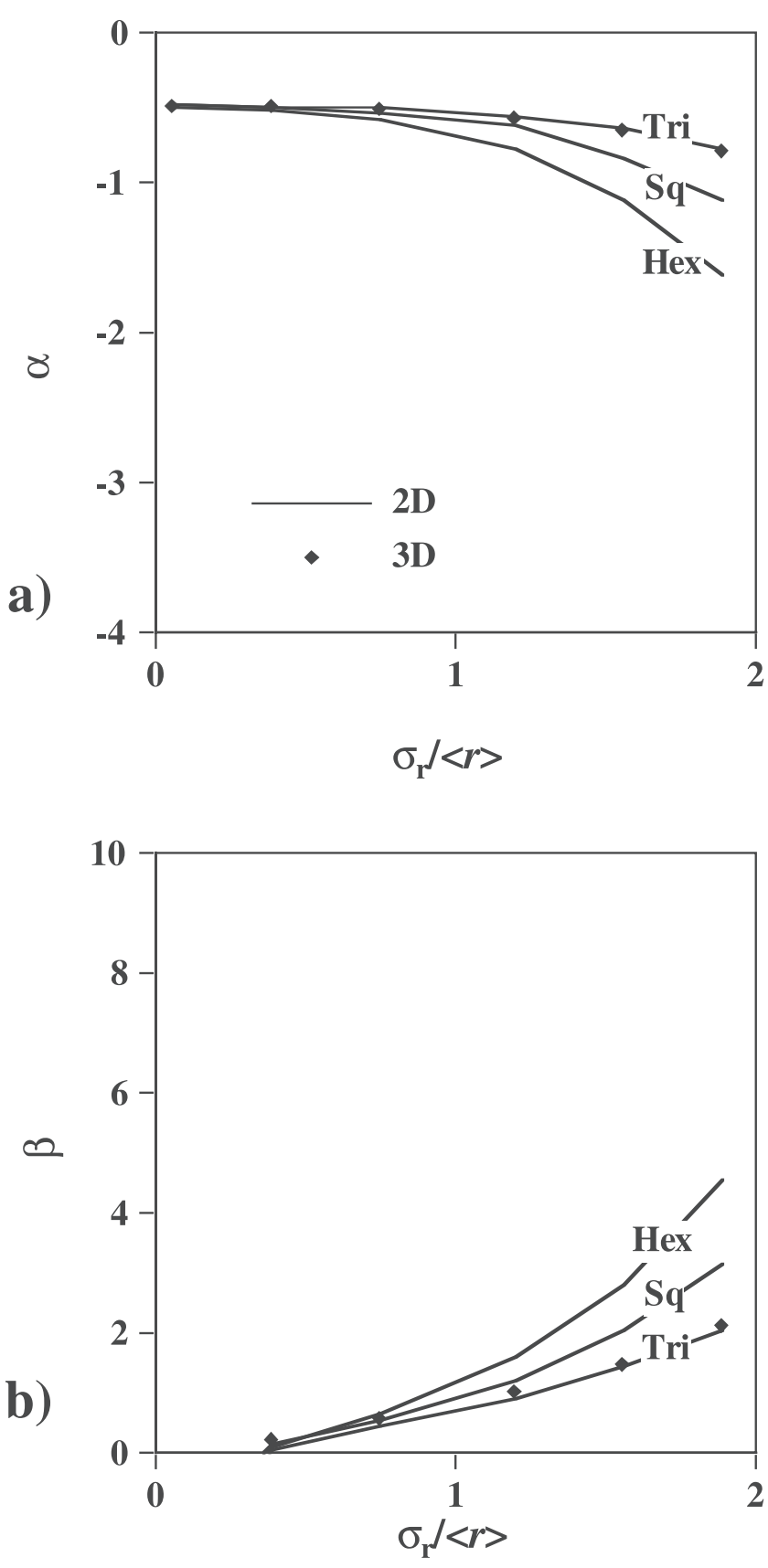

Figure 6. The power law parameters calculated for $\sigma_{1 / F}$ using the $\mathrm{ChD}$ results only (i.e., for networks smaller than $32 \times 32$ or $10 \times 10 \times 10$ ): (a) the exponent $\alpha$ and $(b) \beta$, the decimal $\log$ of the pre-factor. The symbols are the same as in Figure 2.

lack of time, we did not use PBCG in the case of the 2D triangular and hexagonal lattices.

[22] In all three lattices, we observed that $k$ and $1 / F$ did not converge toward unity with increasing network size. In other words, if we estimate $k_{\infty}$ and $1 / F_{\infty}$ as $\langle k\rangle_{g}$ and $\langle 1 / F\rangle_{g}$ for the largest networks considered here, we find that $k_{\infty}$ and $1 / F_{\infty}$ depend on $\sigma_{r} /\langle r\rangle$. This is, of course, expected when the $\mathbf{A}$ distributions are used. Indeed, $k_{\infty}$ and $1 / F_{\infty}$ must increase with increasing $\sigma_{r} /\langle r\rangle$ in the case of the 3D simple cubic and $2 \mathrm{D}$ triangular lattices, whereas the oppo- site has to occur for the 2D hexagonal lattice. The reason is that the upper quartile and the 0.65 quantile of the $\mathbf{A}$ distributions (i.e., critical radius $r_{c}$ of the 3D simple cubic and the 2D triangular lattices, respectively) increase with increasing $\sigma_{r} /\langle r\rangle$ whereas the 0.35 quantile decreases (i.e. $r_{c}$ of the 2D hexagonal lattice). More surprising was the observation that $k_{\infty}$ and $1 / F_{\infty}$ depended on $\sigma_{r} /\langle r\rangle$ even when we used the distributions $\mathbf{B}, \mathbf{C}$ and $\mathbf{D}$, for which the appropriate quantiles were maintained equal to 1 and a behavior similar to that of the 2D square lattice could reasonably be expected. This is illustrated in Figure 7 showing $\langle k\rangle_{a}$ and $\langle k\rangle_{g}$ in $3 \mathrm{D}$ simple cubic networks as a function of $n$. The variations of $k_{\infty}$ with $\sigma_{r} /\langle r\rangle$ appeared to be significant, although much smaller than the variations obtained with the A distributions. They were also quite different in the three lattices considered (see Table 2). The inverse formation factor had essentially the same behavior owing to the approximate relation $k_{\infty} F_{\infty} \approx 1$, as discussed above. One important observation was that, for all lattices, the distributions of $k$ and $1 / F$ obtained with the two types of $r$ distribution used (i.e., $\mathbf{A}$ and $\mathbf{B}, \mathbf{A}$ and $\mathbf{C}$, or $\mathbf{A}$ and $\mathbf{D}$ ) were identical after normalization with respect to $k_{\infty}$ and $1 / F_{\infty}$.

[23] As in the previous section, we observed a strong increase of $\langle k\rangle_{a}$ with decreasing $n$ in all lattices. However, unlike the 2D square lattice, the triangular, hexagonal and cubic lattices did not produce $\langle k\rangle_{g}=1$ independently on $n$ and $\sigma_{r} /\langle r\rangle$. We also observed a decrease of $\langle k\rangle_{g}$ with increasing $n$ and $\sigma_{r} /\langle r\rangle$ in both two-dimensional lattices whereas $\langle k\rangle_{g}$ increased with $n$ in the 3D simple cubic lattice (see Figure 7). The variations of $\langle k\rangle_{g}$ were generally much

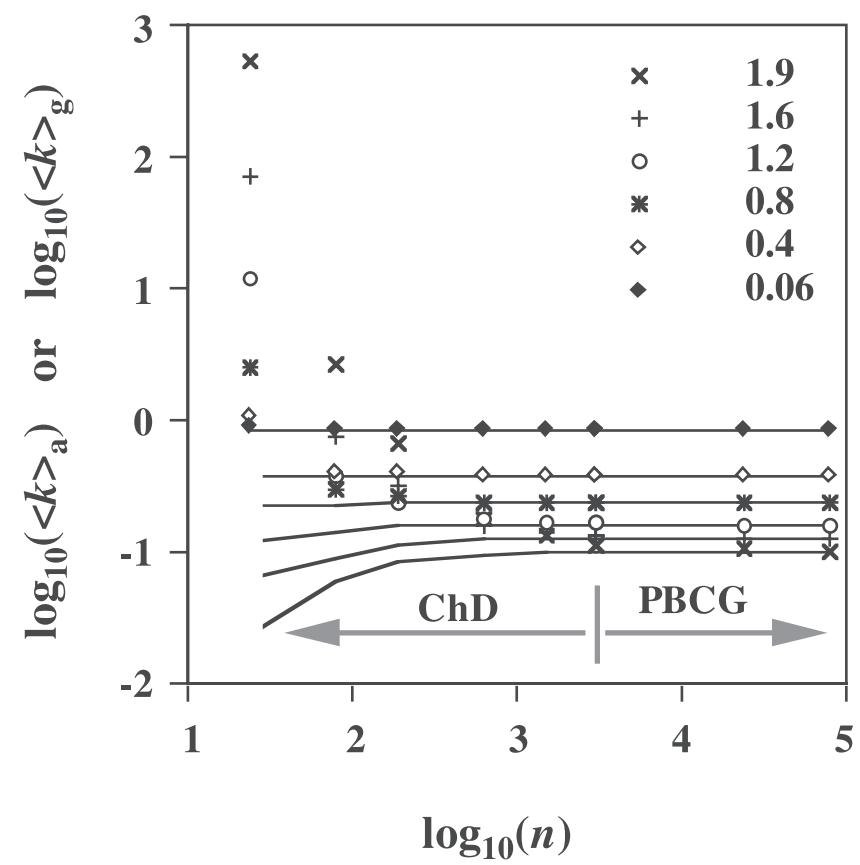

Figure 7. Illustration of the permeability scaling in the case of the 3D simple cubic lattice. Notice the large negative scale dependence of $\langle k\rangle_{a}$ (same symbols as in Figure 1) and the small positive scale dependence of $\langle k\rangle_{g}$ (solid lines). As indicated in the diagram, the ChD solving method was used in networks smaller than $32 \times 32$ while PBCG was utilized above this size. 
Table 2. Values of $k_{\infty}$ and $1 / F_{\infty}$ as a Function of $\sigma_{r} /\langle r\rangle$ for Various Lattices

\begin{tabular}{lcccccc}
\hline & \multicolumn{7}{c}{$\sigma_{r} /\langle r\rangle$} \\
\cline { 2 - 7 } & 0.06 & 0.4 & 0.8 & 1.2 & 1.6 & 1.9 \\
\hline A, 2D square & & & & & & \\
$\quad k_{\infty}$ & 1.0 & 1.0 & 1.0 & 1.0 & 1.0 & 1.0 \\
$\quad 1 / F_{\infty}$ & 1.0 & 1.0 & 1.0 & 1.0 & 1.0 & 1.0 \\
B, 3D simple cubic & & & & & & \\
$\quad k_{\infty}$ & 0.83 & 0.37 & 0.23 & 0.16 & 0.12 & 0.10 \\
$\quad 1 / F_{\infty}$ & 0.91 & 0.56 & 0.37 & 0.25 & 0.20 & 0.16 \\
C, 2D triangular & & & & & & \\
$\quad k_{\infty}$ & 0.90 & 0.63 & 0.57 & 0.59 & 0.61 & 0.65 \\
$\quad 1 / F_{\infty}$ & 0.95 & 0.73 & 0.63 & 0.57 & 0.59 & 0.60 \\
$\mathbf{D}, 2 \mathrm{D}$ hexagonal & & & & & & \\
$\quad k_{\infty}$ & 1.11 & 1.61 & 1.75 & 1.83 & 1.86 & 2.04 \\
$1 / F_{\infty}$ & 1.06 & 1.37 & 1.59 & 1.75 & 1.77 & 1.82 \\
\hline
\end{tabular}

smaller than those of $\langle k\rangle_{a}$, with the largest ones corresponding to the hexagonal lattice.

[24] For all lattices, we performed the power law analysis described in section 3.1, on the normalized $\delta\left(\langle k\rangle_{a}\right)$ and $\delta\left(\langle 1 / F\rangle_{a}\right)$ in networks solved using $\mathrm{ChD}$ (i.e., smaller than $32 \times 32$ or $10 \times 10 \times 10$ ). The values of $\alpha$ and $\beta$ found are plotted in Figures 2 and 3. We see that, for all the lattices, $-\alpha$ and $\beta$ increase with increasing $\sigma_{r} /\langle r\rangle$. The $2 \mathrm{D}$ triangular and 3D simple cubic lattices have the smallest values of $-\alpha$ and $\beta$ whereas the $2 \mathrm{D}$ hexagonal lattice has the largest. The 2D square lattice is in the middle. This order is consistent with the order of the percolation thresholds $(0.25$ for $3 \mathrm{D}$ simple cubic, 0.35 for $2 \mathrm{D}$ triangular, 0.5 for $2 \mathrm{D}$ square and 0.65 for $2 \mathrm{D}$ hexagonal).

[25] The results of the power law analysis of $\sigma_{k}$ and $\sigma_{1 / F}$ are presented in Figures 5 and 6 . As in the last paragraph we see that, for all the lattices, $-\alpha$ and $\beta$ increase with increasing $\sigma_{r} /\langle r\rangle$. Note that the values obtained for the 2D triangular and 3D simple cubic lattices appear to coincide exactly with each other. This is a very surprising result since these two lattices have different dimensionalities. The fact that their only common feature is the coordination number, $Z=6$, suggests that connectivity is the main parameter controlling transport properties scaling in these networks. The 2D triangular and 3D simple cubic lattices have the smallest values of $-\alpha$ and $\beta$, the $2 \mathrm{D}$ hexagonal lattice the largest, and, as before, the 2D square lattice the medium ones. Again, the values of $\sigma_{k}$ obtained for the 3D simple cubic lattice using PBCG (i.e., for networks $10 \times 10 \times 10$ and larger) revealed a transition to the same "universal" power law as before (i.e., $\alpha=-0.5$ ).

\section{Discussion and Conclusions}

[26] The first point to discuss concerns the power law analysis extensively used here. Although the fit was always of good quality (i.e., correlation coefficients larger than 0.95 ), we do not think these power laws can be extrapolated to an infinite range of $n$. Indeed, extrapolating the straight lines in Figure 4a leads to conceptually impossible crossover points. Cross-over cannot occur because the fluctuations of $k$ and $1 / F$ should be strictly monotonic functions of $\sigma_{r} /\langle r\rangle$ for any network size. The PBCG data in Figure 4b confirm that a transition occurs at a sufficiently large network size and suggest that, beyond this transition, the slope of the $\log \left(\sigma_{k}\right)$ versus $\log (n)$ curves approaches a single value, -0.5 , for all values of $\sigma_{r} /\langle r\rangle$ (a similar behavior was observed for the 3D simple cubic lattice). We surmise that this transition to a "universal" scaling behavior is generally valid in all lattices (i.e., independently on topology and dimensionality) and for all parameters studied here, i.e., $\delta(\langle k\rangle), \delta(\langle 1 / F\rangle), \sigma_{k}$ and $\sigma_{1 / F}$. The existence of a transition from non-universal scaling at small scales to universal scaling at large scales is also supported by theoretical arguments based on percolation theory [Hunt, 1998, 2001]. The universal exponent is -1 for $\delta(\langle k\rangle)$ and $\delta(\langle 1 / F\rangle)$ versus $n$, and -0.5 for $\sigma_{k}$ and $\sigma_{1 / F}$ versus $n$. Accordingly, for $\delta(\langle k\rangle)$ and $\delta(\langle 1 / F\rangle)$ versus the linear scale $S$, the universal exponent becomes -2 in two dimensions and -3 in three dimensions (in agreement with the theoretical prediction of Hunt [1998] only in the two-dimensional case), whereas it is equal to -1 in two dimensions and $-3 / 2$ in three dimensions for $\sigma_{k}$ and $\sigma_{1 / F}$ versus $S$. The transition defines a characteristic size reminiscent of the concept of Statistical Representative Elementary Volume (SREV) recently proposed by Zhang et al. [2000] (i.e., in volumes larger than the SREV the transport properties become weakly scale dependent; see also Hunt [1998, 2001]). Our interpretation is that, in networks smaller than the transition size, the fluctuations of $k$ and $1 / F$ are controlled not only by the underlying $r$ distribution but also by the relative positions of small and large pipes. In particular, the occurrence of through-going chains of large pipes must generate extremely large permeability fluctuations. The probability of occurrence of such a chain decreases as its length increases, explaining that the fluctuation enhancement effect described above decreases with increasing network size. According to this explanation, past the transition, the probability of occurrence of through-going chains becomes negligible, the fluctuations of $k$ and $1 / F$ only depend on the underlying $r$ distribution, and a weak scale dependence results (i.e., characterized by low values of $\alpha$ ). Notice also that fluctuation enhancement must obviously increase with increasing heterogeneity as was observed here.

[27] The position of the transition $n^{*}$ can be estimated from $\log _{10}\left(\sigma_{k}\right)$ versus $\log _{10}(n)$ curves (see the examples in Figure 4b). Although precise quantification was not attempted here, we can state that the transition scale $S^{*}$ (i.e., corresponding to $n^{*}$ ) appeared to be a weakly increasing function of heterogeneity [Hunt, 2001]. We find that, in the average, a $30 \times 302 \mathrm{D}$ square network should be an acceptable SREV while we may need networks slightly greater than $10 \times 10 \times 10$ in the case of the 3D simple cubic lattice. Thus $S^{*}$ appeared to be significantly larger than the correlation length (i.e., the length of an individual pipe here). This suggests that the non-universal scaling laws described above may apply in field situations where the system size $S$ is on the order of a few tens of correlation lengths (see also Hunt [2001]).

[28] Since the values of $\alpha$ are much closer to -0.5 for $1 / F$ than $k$, the SREV size appropriate for electrical conduction must be smaller than that defined above for hydraulic flow. This is not surprising. For a given $r$ distribution, the local hydraulic conductances (proportional to $r^{4}$ ) have a much wider distribution than the electrical ones (proportional to $r^{2}$ ). Generally speaking, this study confirms that $1 / F$ has essentially the same properties as $k$, but strongly attenuated. This justifies the numerous attempts of estimating perme- 
ability variations from electrical measurements in the field, but also points out an important practical problem, namely that it is difficult to determine accurately the variations of a very irregular parameter (i.e., $k$ ) from measurements of a much smoother one (i.e., $1 / F)$. The percolation-based relation $r_{c}^{2}=8 k F$ is potentially very useful but an independent technique of estimating $r_{c}$ is then required (remember also that this relation implies that surface electrical conduction is unimportant).

[29] Another related remark is that ensemble geometric averages are much closer to 1 than ensemble arithmetic averages in all lattices (one obtains exactly 1 only for the 2D square lattice). In other words, geometric averaging, in general, yields relatively accurate estimations of $k_{\infty}$. Note however that an overestimation by up to one order of magnitude can occur in 2D hexagonal networks with high $\sigma_{r} /\langle r\rangle$ and low $n$. Also, as explained previously, $1 / F$ is less sharply variable than $k$. Consequently, geometric averaging works even better for $1 / F$, with discrepancies on the order of a few tens of percent (at worst, an overestimation of $1 / F_{\infty}$ by a factor of 2 is produced in 2D hexagonal networks with high $\sigma_{r} /\langle r\rangle$ and low $n$ ).

[30] A second important point is that the large (statistical) increase of $k$ with increasing measurement scale commonly observed in the field [e.g., Brace, 1980; Painter, 1996; Schulze-Makuch et al., 1999] does not occur here (note that we use "size" and "scale" synonymously hereafter). Early studies attributed this large, positive scale dependence to a bias in the sampling of the permeability distribution at small scales (for example, fractures are systematically excluded in laboratory measurements, Brace [1980]). In that case, a positive scale effect is an artifact, not a property of the geological systems considered. More recent explanations are based on assuming nested heterogeneity structures [e.g., Di Federico and Neuman, 1997]. However, the effect of nested structures is somewhat controversial. In a recent theoretical discussion, Hunt [2003] reported an example of nested structure producing a negative scale effect. He also speculated that nested structures should not lead to positive scale effects unless they involve some sort of sampling bias. A related work is that of de Dreuzy et al. [2001a, 2001b], who studied flow through two-dimensional, random fracture networks obeying power law length distributions. Although these authors do not use the term "nested structure," their fracture networks provide an excellent example of hierarchical media. Interestingly, they were able to vary the hierarchiness continuously by changing the exponent $a$ of the fracture length distribution. For $a<3$ long fractures are present (including fractures longer than the network considered), the fracture network is significantly hierarchical, and a positive scale effect was found. This result may provide a clue to clarify the relation between positive scale effects and sampling biases. Indeed, in a flow measurement on a given network, the fractures longer than the network scale are obviously undersampled. It is revealing that de Dreuzy et al. [2001b] observed unlimited scale effects only for $a<2$ (i.e., very strong nested structure) and a perfect correlation between length and aperture of individual fractures, that is, when the undersampling of high permeabilities at any given scale was the most severe. For $a>3$ long fractures are essentially absent, the fracture network has a weak nested structure, and no positive scale effects were found. This is in qualitative agreement with our results since our pipe networks were not hierarchical and sampling biases were forbidden by our procedures.

[31] Whatever the importance of nested structures in geologic materials, its effect must decrease and eventually become negligible if the maximum scale considered is reduced. Accordingly, we expect that nested structures should be largely unimportant in the samples or blocks used in the laboratory studies of Henriette et al. [1989], Tidwell and Wilson [1997, 1999a, 1999b, 2000], and Zhang et al. [2000]. Consistently with our results, Zhang et al. [2000] did find that $\langle k\rangle_{a}$ decreases and $\sigma_{k}$ increases with increasing measurement scale. They also observed an increase of $\sigma_{\Phi}$ (and, strangely, of $\langle\Phi\rangle_{a}$ ), but it is visually clear that the rate of change is lower for $\sigma_{\Phi}$ than for $\sigma_{k}$. From the values reported in Henriette et al. [1989] we estimated $\langle k\rangle_{a}$, $\langle k\rangle_{g}, \sigma_{k}$ and $\sigma_{\Phi}$ for two different scales $\left(\langle\Phi\rangle_{a}\right.$ was also calculated and found constant as it should be). In agreement with our network results, we observed an increase of $\sigma_{k}$ and $\sigma_{\Phi}$, a decrease of $\langle k\rangle_{a}$, and, more importantly, an increase of $\langle k\rangle_{g}$ with increasing scale as in the $3 \mathrm{D}$ simple cubic networks. Note however that the variations appear to be rather small. Again we observe that the rate of change seems to be lower for $\sigma_{\Phi}$ than for $\sigma_{k}$. Following Henriette et al. [1989] we constructed very large network realizations $(96 \times 96$ and $32 \times 32 \times 32$ ), divided them in smaller and smaller subnetworks, calculated $k$ in all networks and subnetworks (using PBCG when needed) and examined the variations of $k$ as a function of size. The $k$ and $1 / F$ distributions obtained with this new procedure were not distinguishable from the ones described in the previous sections. This is not surprising since ergodicity is implied in our procedure.

[32] In a series of thorough studies, Tidwell and Wilson [1997, 1999a, 1999b, 2000] systematically quantified the spatial fluctuations of permeability in meter-scale blocks of sandstones and volcanic tuff as a function of $R$, the radius of the measuring device. In all cases they found that the variance of $\log (k)$ was a decreasing power law of $R$ with an exponent varying between -0.3 and -0.6 (note that these values cannot be directly compared to ours). They also calculated $\langle\log (k)\rangle_{a}$ (i.e., equivalent to the geometric average $\langle k\rangle_{g}$ ) and discovered that it increased with increasing $R$ in the sandstone blocks while it decreased in the volcanic tuff block. The measured variations were significant (for an approximately 8.5 -fold increase in $R,\langle k\rangle_{g}$ increased by a factor of 1.5 in Berea sandstone, 2.2 in Massillon sandstone and decreased by a factor of 1.5 in volcanic tuff). Interestingly, $\langle k\rangle_{a}$ had the same behavior as $\langle k\rangle_{g}$ (that is, it increased with increasing $R$ in the sandstone blocks while it decreased in the volcanic tuff block).

[33] The negative scale dependence of $\langle k\rangle_{g}$ in volcanic tuff is not consistent with our results for the $3 \mathrm{D}$ simple cubic lattice but it is possible that the pore space of volcanic tuff is better represented by some other, differently behaving, 3D lattice than by the simple cubic lattice. On the other hand, the positive scale dependence of $\langle k\rangle_{a}$ in the two sandstones is in serious disagreement with our results. We argue however that the results of Tidwell and Wilson [1997, 1999a, 2000] are faulty despite the outstanding carefulness of their work. Our opinion is based on the following reasoning: assuming that the distribution of permeability 
at the smallest scale $S_{0}$ is completely known and that it is bounded by $k_{\min }$ and $k_{\max }$ (a reasonable assumption since the system considered is not infinite), the permeability of any sample of volume larger than $S_{0}$ must be lower (respectively, greater) than $k_{\max }$ (respectively, $k_{\min }$ ). Note that this must be true whether the spatial distribution of $k$ is correlated or not. The extrema are actually reached only in homogeneous volumes of material with $k=k_{\max }\left(\right.$ or $\left.k_{\min }\right)$ at every point. Consequently, the successive $k$ distributions at increasing scales must be entirely included within each other (i.e., nested distributions) as illustrated in Figure 8a (showing the $k$ distributions obtained here in 2D square networks of size $4 \times 4,8 \times 8,16 \times 16$ and $32 \times 32$ with distribution A4). The $k$ distributions reported in Tidwell and Wilson [1997, 1999a, 2000] were not nested (they were similar to the distributions illustrated in Figure 8c), implying that the measurements of $k$ with the smallest $R$ used in these studies were statistically biased. Notice that the absence of this anomaly in the case of volcanic tuff coincided with a negative scale dependence of $\langle k\rangle_{a}$. It seems likely to us that the anomalous behavior of the sandstone blocks is related to the rock rather than to the experimental method. Perhaps, quarrying produced a thin damaged layer at the surface of relatively hard rocks like Berea and Massillon sandstones whereas this did not occur in softer volcanic tuff. Alternatively, salt precipitation due to weathering may reduce permeability inside a thin layer at the rock surface [e.g., Evans, 1969; Jeannette, 2000]. Note that the volcanic tuff block was indeed cut very shortly before the permeability measurements were made (no such information was specified for the sandstone blocks).

[34] Following Hunt [1998, 2001, 2003], we define the scaling problem as follows: knowing the complete distribution of some property $X\left(\right.$ e.g., $k$ ) at a minimum scale $S_{0}$ (i.e., $X$ measured in rock volumes of size $S_{0}$ covering the entire system considered), what are the distributions of $X$ at scales larger than $S_{0}$ ? An increase of the ensemble mean of $X$ with increasing scale is called a "positive scale effect" (PSE). It is necessary to further distinguish between "weak" and "strong" PSE. Weak PSE corresponds to the case where the distributions of $X$ at increasing scales are nested (consistently with the assumption of absence of sampling bias at the minimum scale) and the ensemble mean of $X$ nevertheless increases with scale. This can be achieved as illustrated in Figure $8 \mathrm{~b}$ where the successive distributions all have an approximately identical maximum, forcing the ensemble mean to increase with scale. Strong PSE denotes the case illustrated in Figure 8c. The successive distributions are not nested, with their maxima increasing with scale. For most physical properties of rocks (e.g., $k$ ), strong PSE indicates sampling bias at small scales. As discussed above, the data of Tidwell and Wilson [1997, 1999a, 2000] showed strong PSE, and so did the field studies reported by Brace [1980] or Schulze-Makuch et al. [1999]. The results of de Dreuzy et al. [2001a, 2001b] seem to belong to the weak PSE class despite the large amplitude of the PSE reported, which is simply due to the fact that $k_{\max }$ is huge in the case of large fractures. Our results for $\langle k\rangle_{g}$ indeed showed that weak PSE is not strictly impossible. Weak PSE should be favored if the high-permeability elementary volumes have enhanced connectivity (e.g., fractures). Our contention is again that these conclusions should generally hold in macroscopic a)
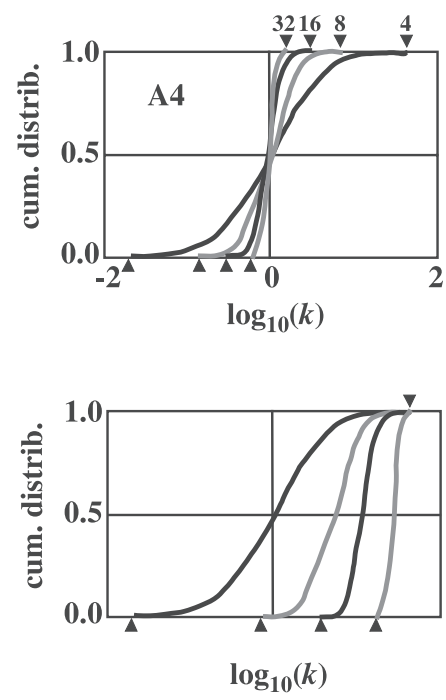

c)

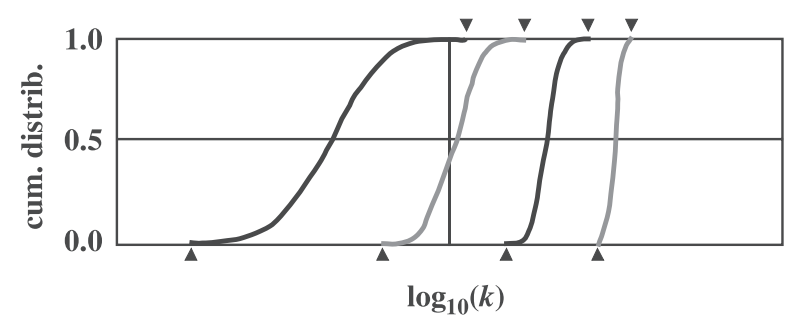

Figure 8. (a) Example of ensemble cumulative distributions of $\log _{10}(k)$ obtained at different scales (i.e., in $4 \times 4$, $8 \times 8,16 \times 16$, and $32 \times 32$ networks) for the 2 D square lattice with $r$ distribution A4. These distributions all appear symmetric, suggesting a lognormal character. Note also that they all intersect each other at the median point corresponding to $k=1$. This is consistent with our observation that $\langle k\rangle_{g}$ is always equal to unity in the $2 \mathrm{D}$ square lattice. The successive minima and maxima are indicated by solid triangles, demonstrating that these distributions were nested. (b) Illustration of the "weak" PSE. The successive distributions are nested and have the same maximum. The successive mean values must increase with scale, eventually reach the maximum asymptotically at infinite scales. (c) Illustration of the "strong" PSE. The successive distributions are not nested, indicating that sampling bias occurred at small scales.

porous media at scales larger than the correlation length [Hunt, 1998].

[35] We also demonstrated that, in two-dimensional lattices, the fluctuations of $k$ and $1 / F$ and, more importantly, their scaling properties depend on topology. From our results, it appears that the most appropriate topological parameter to consider is the lattice coordination number $Z$ (i.e., number of connected bonds per node). Remembering that $Z$ characterizes the lattice connectiveness, we see that, in twodimensional lattices, the less connected the lattice the greater the fluctuation enhancement effect described in section 3 . We can reasonably assume that this is also true in three-dimensional lattices and in the real pore networks of rocks. New imaging technologies now allow acquisition of high-resolution three-dimensional images of the pore 
space of rocks [e.g., Spanne et al., 1994; Fredrich et al., 1995]. Using the medial axis analysis, Lindquist et al. [1996] showed that the pore space of rocks can be represented as a graph, the topology of which can be quantified. Recently, Lindquist et al. [2000] showed that the pore network of Fontainebleau sandstone had locally measured coordination numbers strongly fluctuating in space around an average value between 3 and 4 . This is significantly lower than 6 , the value corresponding to the $3 \mathrm{D}$ simple cubic lattice. Hence we expect the overall percolation threshold in this type of rock (i.e., clean sandstone) to be higher than 0.25 (maybe to approach 0.5 ), therefore yielding scaling properties more resembling the $2 \mathrm{D}$ square lattice than the 2D triangular lattice. Low values of the coordination number are also consistent with a clearly identifiable SREV as observed by Zhang et al. [2000].

[36] It is well known that the network flow equations are mathematically similar to the finite difference equations of Darcian flow. Our results can therefore be generalized to numerical simulations of flow through macroscopically heterogeneous porous media. In particular, the topology of the grid used should affect the results (e.g., triangular grids are known to reduce grid-orientation effects [Mattax and Dalton, 1990]). This remark is especially important if the goal of the numerical simulations is to establish a scaling rule. In two-dimensional models we stress again that the widely used square grid has unique characteristics and may not yield truly representative results in systems with a number of grid blocks insufficiently large. Of course, these problems should vanish in systems with a very large number of grid blocks since scaling becomes presumably "universal."

[37] Acknowledgments. We are grateful to Allen Hunt and the Associate Editor, Mickael Manga, for helping us improve this paper. Most of this work was performed during YB's visits to MIT and was partially funded by the US Department of Energy under grant DE-FG02-00ER.

\section{References}

Ababou, R., D. McLaughlin, L. W. Gelhar, and A. F. B. Tompson, Numerical simulation of three-dimensional saturated flow in randomly heterogeneous porous media, Transp. Porous Media, 4, 549-565, 1989.

Ambegaokar, V. N., B. I. Halperin, and J. S. Langer, Hopping conductivity in disordered systems, Phys. Rev. B, 4, 2612-2620, 1971.

Bernabé, Y., The frequency dependence of harmonic fluid flow through networks of cracks and pores, Pure Appl. Geophys., 149, 489-506, 1997.

Bernabé, Y., and C. Bruderer, Effect of the variance of pore size distribution on the transport properties of heterogeneous networks, J. Geophys. Res., 103, 513-525, 1998.

Brace, W. F., Permeability of crystalline and argillaceous rocks, Int. J. Rock Mech. Min. Sci. Geomech. Abstr., 17, 241-251, 1980.

Brown, G. O., H. T. Hsieh, and D. A. Lucero, Evaluation of laboratory dolomite core sample size using representative elementary volume concepts, Water Resour. Res., 36, 1199-1207, 2000.

Bruderer, C., and Y. Bernabé, Network modeling of dispersion: Transition from Taylor dispersion in homogeneous networks to mechanical dispersion in very heterogeneous ones, Water Resour. Res., 37, 897-908, 2001.

Cushman, J. H., On measurement, scale, and scaling, Water Resour. Res., 22, 129-134, 1986

Dagan, G., Statistical theory of groundwater flow and transport: Pore to laboratory, laboratory to formation, and formation to regional scale, Water Resour. Res., 22, 120S-134S, 1986.

de Dreuzy, J.-R., P. Davy, and O. Bour, Hydraulic properties of two-dimensional random fracture networks following a power law length distribution: 1. Effective connectivity, Water Resour. Res., 37, 2065-2078, 2001a.

de Dreuzy, J.-R., P. Davy, and O. Bour, Hydraulic properties of two-dimensional random fracture networks following a power law length distribution: 2. Permeability of networks based on lognormal distribution of apertures, Water Resour. Res., 37, 2079-2095, 2001 b.
Di Federico, V., and S. P. Neuman, Scaling of random fields by means of truncated power variograms and associated spectra, Water Resour. Res., 33, 1075-1085, 1997.

Durlofsky, L. J., Numerical calculation of equivalent grid block permeability tensors for heterogeneous porous media, Water Resour. Res., 27, 699708,1991

Durlofsky, L. J., Representation of grid block permeability in coarse scale models of randomly heterogeneous porous media, Water Resour. Res., 28, $1791-1800,1992$.

Evans, I. S., Salt crystallization and rock weathering: A review, Rev. Geomorph. Dyn., 4, 153-177, 1969.

Fredrich, J. T., and W. B. Lindquist, Statistical characterization of the threedimensional microgeometry of porous media and correlation with macroscopic transport properties, Int. J. Rock Mech. Min. Sci. Geomech. Abstr., 34, paper 085, 1997.

Fredrich, J. T., B. Menendez, and T.-F. Wong, Imaging the pore structure of geomaterials, Science, 268, 276-279, 1995.

Friedman, S. P., and N. A. Seaton, Critical path analysis of the relationship between permeability and electrical conductivity of three-dimensional pore networks, Water Resour. Res., 34, 1703-1710, 1998.

Garbesi, K., R. G. Sextro, A. L. Robinson, J. D. Wooley, J. A. Owens, and W. W. Nazaroff, Scale dependence of soil permeability to air: Measurement method and field investigation, Water Resour. Res., 32, 547-560, 1996.

Gelhar, L. W., Stochastic Subsurface Hydrology, 390 pp., Prentice-Hall, Old Tappan, N. J., 1993.

Gelhar, L. W., and C. L. Axness, Three-dimensional stochastic analysis of macrodispersion in aquifers, Water Resour. Res., 19, 161-180, 1983.

Goggin, D. J., M. A. Chandler, G. Kocurek, and L. W. Lake, Permeability transects of eolian sands and their use in generating random permeability fields, SPE Form. Eval., 7, 7-16, 1992.

Henriette, A., C. G. Jacquin, and P. M. Adler, The effective permeability of heterogeneous porous media, PCH PhysicoChem. Hydrodyn., 11, 63-80, 1989.

Hunt, A. G., Upscaling in subsurface transport using cluster statistics of percolation, Transp. Porous Media, 30, 177-198, 1998.

Hunt, A. G., Applications of percolation theory to porous media with distributed local conductances, Adv. Water Resour., 24, 279-307, 2001.

Hunt, A. G., Some comments on the scale dependence of the hydraulic conductivity in the presence of nested heterogeneity, Adv. Water Resour., 26, 71-77, 2003.

Jeannette, D., Originalité des mécanismes d'altération sur les vestiges archéologiques de Delos (Cyclades Grèce), C.R. Acad. Sci., Ser. II, $330,683-688,2000$.

Lindquist, W. B., S.-M. Lee, D. A. Coker, K. W. Jones, and P. Spanne, Medial axis analysis of void structure in three-dimensional tomographic images of porous media, J. Geophys. Res., 101, 8297-8310, 1996.

Lindquist, W. B., A. Venkatarangan, J. Dunsmuir, and T.-F. Wong, Pore and throat size distributions measured from synchrotron X-ray tomographic images of Fontainebleau sandstones, J. Geophys. Res., 105, 21,50921,527, 2000.

Mattax, C. C., and R. L. Dalton, Reservoir Simulation, SPE Monogr., vol. 13, 173 pp., Soc. Of Pet. Eng., Richardson, Tex., 1990.

Netto, A. S. T., Pore-size distribution in sandstones, AAPG Bull., 77, $1101-$ 1104, 1993.

Newman, S. P., Generalized scaling of permeabilities: Validation and effect of support scale, Geophys. Res. Lett., 21, 349-352, 1994.

Painter, S., Evidence for non-Gaussian scaling behavior in heterogeneous sedimentary formations, Water Resour. Res., 32, 1183-1195, 1996.

Pollak, M. A., Percolation treatment of dc hopping conduction, J. Non Cryst. Solids, 11, 1-24, 1972.

Press, W. H., S. A. Teukolsky, W. T. Vetterling, and B. P. Flannery, Numerical Recipes in C: The Art of Scientific Computing, 2nd. ed., 994 pp., Cambridge Univ. Press, New York, 1992.

Purvance, D. T., and R. Andricevic, On the electrical-hydraulic conductivity correlation in aquifers, Water Resour. Res., 36, 2905-2913, 2000a.

Purvance, D. T., and R. Andricevic, Geoelectric characterization of the hydraulic conductivity field and its spatial structure at variable scales, Water Resour. Res., 36, 2915-2924, 2000b.

Renard, P., and G. de Marsily, Calculating equivalent permeability: A review, Adv. Water Resour., 20, 253-278, 1997.

Sahimi, M., Flow and Transport in Porous Media and Fractured Rock, 482 pp., John Wiley, Hoboken, N. J., 1995.

Schulze-Makuch, D., D. A. Carlson, D. S. Cherkauer, and P. Malik, Scale dependency of hydraulic conductivity in heterogeneous media, Ground Water, 37, 904-919, 1999

Spanne, P., J. Thovert, C. Jacquin, W. B. Lindquist, K. Jones, and P. Adler, Synchrotron computed microtomography of porous media: Topology and transports, Phys. Rev. Lett., 73, 2001-2004, 1994.

Stauffer, D., and A. Aharony, Introduction to Percolation Theory, 2nd. ed., 180 pp., Taylor and Francis, Washington, D. C., 1992. 
Tidwell, V. C., and J. L. Wilson, Laboratory method for investigating permeability upscaling, Water Resour. Res., 33, 1607-1616, 1997.

Tidwell, V. C., and J. L. Wilson, Permeability upscaling measured on a block of Berea sandstone: Results and interpretation, Math Geol., 31, 749-769, 1999a.

Tidwell, V. C., and J. L. Wilson, Upscaling experiments conducted on a block of volcanic tuff: Results for a bimodal permeability distribution, Water Resour. Res., 35, 3375-3387, 1999b.

Tidwell, V. C., and J. L. Wilson, Heterogeneity, permeability patterns, and permeability upscaling: Physical characterization of a block of Massilon sandstone exhibiting nested scales of heterogeneity, SPE Reservoir Eng. Eval. Eng., 3, 283-291, 2000

Wong, T.-F., J. Fredrich, and G. D. Gwanmesia, Crack aperture statistics and pore space fractal geometry of Westerly granite and Rutland quartzite: Implications for elastic contact models of rock compressibility, J. Geophys. Res., 94, 10,267-10,278, 1989.
Zhang, D., R. Zhang, S. Chen, and W. E. Soll, Pore scale study of flow in porous media: Scale dependency, REV, and statistical REV, Geophys. Res. Lett., 27, 1195-1198, 2000.

Zhu, W., and T.-F. Wong, Network modeling of the evolution permeability and dilatancy in compact rock, J. Geophys. Res., 104, 2963-2971, 1999.

Y. Bernabé and A. Maineult, Institut de Physique du Globe, Université Louis Pasteur, Centre National de Recherche Scientifique, 5 rue Rene Descartes, F-67084 Strasbourg, France. (ybernabe@eost.u-strasbg.fr; Alexis.Maineult@eost.u-strasbg.fr)

C. Bruderer-Weng, Hydrosciences, Institut des Sciences de la Terre, de l'Environnement et de l'Espace de Montpellier, Université de Montpellier 2, F-34095 Montpellier Cedex 5, France. (bruderer@msem.univ-montp2.fr) 